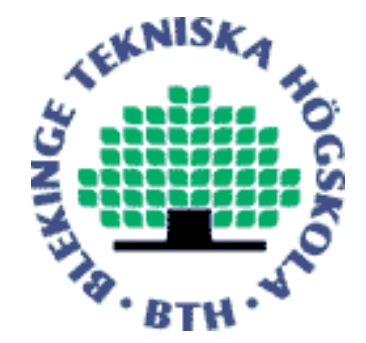

Copyright (C) 2012 IEEE.

Citation for the published paper:

Amplify-and-Forward Relay Assisting both Primary and Secondary Transmissions in Cognitive Radio Networks over Nakagami-m Fading

Thi My Chinh Chu, Hoc Phan, Hans-Jürgen Zepernick

IEEE International Symposium on Personal, Indoor and Mobile Radio Communications (PIMRC)

2012 Sydney, Australia

This material is posted here with permission of the IEEE. Such permission of the IEEE does not in any way imply IEEE endorsement of any of BTH's products or services Internal or personal use of this material is permitted. However, permission to reprint/republish this material for advertising or promotional purposes or for creating new collective works for resale or redistribution must be obtained from the IEEE by sending a blank email message to pubs-permissions@iee.org.

By choosing to view this document, you agree to all provisions of the copyright laws protecting it. 


\title{
Amplify-and-Forward Relay Assisting both Primary and Secondary Transmissions in Cognitive Radio Networks over Nakagami-m Fading
}

\author{
Thi My Chinh Chu, Hoc Phan, and Hans-Jürgen Zepernick \\ Blekinge Institute of Technology, SE-371 79 Karlskrona, Sweden \\ E-mail: $\{$ cch, hph, hjz $\} @$ bth.se
}

\begin{abstract}
In this paper, we study the performance for the primary and secondary transmissions in cognitive radio networks where the amplify-and-forward (AF) secondary relay helps to transmit the signals for both the primary and secondary transmitters over independent Nakagami- $m$ fading. First, we derive exact closed-form expressions for outage probability and symbol error rate (SER) of the primary network. Then, we derive an exact closed-form expression for outage probability and a closed-form expression of a tight upper bound for SER of the secondary network. Furthermore, we also make a comparison for the performance of the primary system with and without the help of the secondary relay. Finally, we show a good agreement between analytical results and Monte-Carlo simulations.
\end{abstract}

\section{INTRODUCTION}

The concept of cognitive relay transmission has been of great interest in the research community thanks to its advantageous features such as efficient utilization of frequency spectrum, reliable transmission, and radio coverage (see [1]-[5] for some recent studies, and the references therein). In particular, a brief overview about techniques for cooperative transmission in a cognitive radio network (CRN) was presented in [1], [2]. Based on the manner that secondary users access the licensed spectrum, there exist overlay cognitive and underlay cognitive spectrum sharing. Under the overlay paradigm, the secondary user is only allowed to use the licensed spectrum of the primary user when this spectrum is not occupied. To opportunistically utilize the licensed frequency, the secondary users adopt a specific spectrum sensing mechanism to decide whether the licensed spectrum is idle. On the contrary, under the underlay scheme, the primary and secondary users may access the same spectrum simultaneously provided that the interference incurred by the secondary transmission at the primary receiver remains below a pre-defined threshold. Consequently, the secondary transmitters must control its transmit power to meet the interference constraint at the primary receiver even if the primary transmitter is idle. For example, the studies of [3] have presented several spectrum sensing techniques for an overlay CRN. Differently, [4] has proposed a distributed power allocation strategy for underlay cognitive multiple-relay network to guarantee the interference contraint.

The two fundamental relaying protocols in conventional relay networks, namely amplify-and-forward (AF) and decodeand-forward (DF), have been also considered in cognitive relay systems. That is, in the works of [6], [7], outage performance of a cognitive AF network with single and multiple relay(s) has been addressed, respectively. In addition, outage probability of a cognitive DF relay network has been quantified in [5], [8]. Nevertheless, all of the aforementioned works have studied the system performance in the context that the relays only assist the transmission of the secondary network rather than the primary user. Thanks to the broadcast nature of wireless communications, theoretically, a secondary relay can receive signals from any neighbor transmitter, including both primary and secondary transmitters. Assisting both the primary and secondary transmissions, the secondary relay can be more helpful in the sense that the reliability of the primary receiver is enhanced. In addition, the above relay assisted infrastructure becomes more efficient in utilizing spectrum because of its high performance. To achieve this benefit, [9] investigates outage probability (OP) for a DF relay transmission in underlay cognitive scheme where a relay retransmits signals for both the primary transmission and the secondary transmission.

In this paper, we study the performance of the primary and secondary transmission in an CRN. We focuses on the scenario that the secondary relay operates in AF mode and is responsible to forward both the primary and secondary signals. In particular, closed-form expressions for the OP and symbol error rate (SER) of both the primary and secondary transmissions are derived to quantify and study the effect of the network parameters on the system performance. Numerical examples are conducted to validate the presented analysis.

The structure of this paper is organized as follows. Section II describes the system model and related definitions. Section III analyzes the OP and SER for both the primary and secondary transmissions. Section IV provides numerical results, discussion and evaluation about the results. Finally, conclusions are presented in Section V.

Notation: The following notations are used in this paper. A vector and a matrix are denoted by bold lower and upper case letters, respectively. Further, $f_{X}(\cdot)$ and $F_{X}(\cdot)$, respectively, stand for the probability density function (PDF) and the cumulative distribution function (CDF) of a random variable (RV) $X$. Additionally, expectation operator is denoted by $\mathbb{E}\{\cdot\}$ and $\mathcal{C N}\left(0, N_{0}\right)$ represents an additive white Gaussian noise (AWGN) RV with zero mean and variance $N_{0}$. We use $\Gamma(n)$ as the gamma function defined in [10, eq. 8.310.1] and $\Gamma(n, x)$ as the incomplete gamma function defined in [10, eq. 8.350.2]. Moreover, the $n$th order modified Bessel function of the second kind in [10, eq. (8.432.1)] and the Whittaker function in [10, eq. (9.222)] are denoted as $\mathcal{K}_{n}(\cdot)$ and $\mathcal{W}_{\lambda, \mu}(x)$, respectively. Finally, $U(a, b ; x)$ is the confluent hypergeometric function [10, eq. (9.211.4)] and ${ }_{2} F_{1}(a, b ; c ; x)$ denotes the Gauss hypergeometric function defined in $[10$, eq. (9.111)].

\section{SySTEM AND CHANNEL MODEL}

We consider a $\mathrm{CRN}$ consisting of a primary transmitter, $\mathrm{PU}_{\mathrm{TX}}$, a primary receiver, $\mathrm{PU}_{\mathrm{RX}}$, a secondary transmitter, $\mathrm{SU}_{\mathrm{TX}}$, a secondary relay, $\mathrm{SU}_{\mathrm{R}}$, and a secondary receiver, $\mathrm{SU}_{\mathrm{RX}}$ 
operating according to the underlay scheme as shown in Fig. 1. We assume $\mathrm{SU}_{\mathrm{TX}}$ is located far from $\mathrm{PU}_{\mathrm{RX}}$ and its average transmit power per symbol is controlled based on the average channel gain from $\mathrm{SU}_{\mathrm{TX}}$ to $\mathrm{PU}_{\mathrm{RX}}$ to meet the interference constraint at $\mathrm{PU}_{\mathrm{RX}}$. Furthermore, due to the long distance and shadowing between $\mathrm{SU}_{\mathrm{TX}}$ and $\mathrm{SU}_{\mathrm{RX}}$, the direct communication from $S_{T X}$ to $S_{R X}$ is not applicable. Therefore, the secondary network uses a relay $\mathrm{SU}_{\mathrm{R}}$ to forward the signals. The secondary network co-exists with the primary network utilizing the frequency band licensed to the primary user by applying underlay spectrum sharing. On the other hand, the secondary relay will support both networks in forwarding their signals. Supposing that all channels are modeled as Nakagami$m$ fading with fading severity parameter $m$ and all terminals operate in half-duplex mode.

In the first time slot, both $\mathrm{PU}_{\mathrm{TX}}$ and $\mathrm{SU}_{\mathrm{TX}}$ simultaneously broadcast their signals, namely $x_{s}$ and $x_{p}$ with average transmit power $P_{p}$ and $P_{s}$, respectively. Hence, the received signals $y_{r}$ at $\mathrm{SU}_{\mathrm{R}}$ and $y_{\mathrm{PD}}$ at $\mathrm{PU}_{\mathrm{RX}}$ are, respectively, given by

$$
\begin{aligned}
y_{r} & =h_{1} x_{s}+h_{3} x_{p}+n_{r} \\
y_{\mathrm{PD}} & =h_{5} x_{p}+n_{p}
\end{aligned}
$$

where $h_{1}, h_{3}, h_{5}$ are the channel coefficients of the links $\mathrm{SU}_{\mathrm{TX}} \rightarrow \mathrm{SU}_{\mathrm{R}}, \mathrm{PU}_{\mathrm{TX}} \rightarrow \mathrm{SU}_{\mathrm{R}}$, and $\mathrm{PU}_{\mathrm{TX}} \rightarrow \mathrm{PU}_{\mathrm{RX}}$, respectively. In addition, $n_{r}, n_{p}$ are the additive white Gaussian noise (AWGN) with zero mean and variance $N_{0}$ at $\mathrm{SU}_{\mathrm{R}}$ and $\mathrm{PU}_{\mathrm{RX}}$, respectively. Note that $h_{3} x_{p}$ now becomes the interference component for the secondary transmission, and $h_{1} x_{s}$ is the interference to the primary transmission. These components are assumed to be large as compared to the noise at $\mathrm{SU}_{\mathrm{R}}$ such that this noise in (1) can be neglected.

In the second time slot, $\mathrm{SU}_{\mathrm{R}}$ amplifies the received signal, $y_{r}$, with amplifying gain $G$ and then forwards the resulting signal. Therefore, the received signals at $\mathrm{PU}_{\mathrm{RX}}$ and $\mathrm{SU}_{\mathrm{RX}}$ are, respectively, expressed as

$$
\begin{aligned}
y_{\mathrm{PR}} & =G h_{4} h_{1} x_{s}+G h_{4} h_{3} x_{p}+n_{p} \\
y_{S} & =G h_{2} h_{1} x_{s}+G h_{2} h_{3} x_{p}+n_{s}
\end{aligned}
$$

where $h_{2}$ and $h_{4}$ are the channel coefficients of the links $\mathrm{SU}_{\mathrm{R}} \rightarrow \mathrm{SU}_{\mathrm{RX}}$ and $\mathrm{SU}_{\mathrm{R}} \rightarrow \mathrm{PU}_{\mathrm{RX}}$, respectively. Moreover, $n_{s}$ is the AWGN at $\mathrm{SU}_{\mathrm{RX}}$ with zero mean and variance $N_{0}$.

The amplifying gain $G$ is selected to meet the interference power constraint at $\mathrm{PU}_{\mathrm{RX}}$. That is, the interference from the secondary transmission, imposed on $\mathrm{PU}_{\mathrm{RX}}$, must be limited to a predefined threshold $Q$, i.e. $\mathbb{E}\left\{\left(G h_{1} x_{s}\right)^{2}\right\}=Q / h_{4}^{2}$ or

$$
G^{2}=\frac{Q}{P_{s} h_{1}^{2} h_{4}^{2}}
$$

As a result, the instantaneous signal-to-interference plus noise ratio (SINR) at $\mathrm{SU}_{\mathrm{RX}}, \gamma_{S}$, is represented as

$$
\gamma_{s}=\frac{X_{1} X_{2}}{a X_{2} X_{3}+b X_{1} X_{4}}
$$

Similarly, the instantaneous SINR of the relaying link, $\gamma_{\mathrm{PR}}$, and the instantaneous signal-to-noise ratio (SNR) of the direct link, $\gamma_{\mathrm{PD}}$, at $\mathrm{PU}_{\mathrm{RX}}$ are, respectively, given by

$$
\begin{aligned}
\gamma_{\mathrm{PR}} & =\frac{X_{3}}{c X_{1}} \\
\gamma_{\mathrm{PD}} & =d X_{5}
\end{aligned}
$$

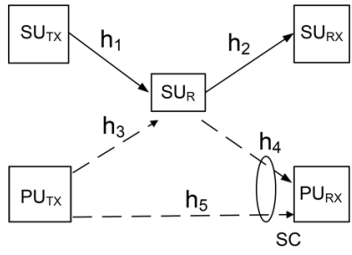

Fig. 1. System model for cognitive AF relay networks.

where $a=P_{p} / P_{s}, b=N_{0} / Q, c=\left(Q+N_{0}\right) P_{s} /\left(Q P_{p}\right), d=$ $P_{p} / N_{0}$ and $X_{l}=h_{l}^{2}$ with $l \in\{1, \ldots, 5\}$. It is assumed that $\mathrm{PU}_{\mathrm{RX}}$ adopts selection combining (SC) to process the received signals. Therefore, its instantaneous end-to-end SNR at $\mathrm{PU}_{\mathrm{RX}}$, $\gamma_{P}$, is written as $\gamma_{P}=\max \left(\gamma_{\mathrm{PR}}, \gamma_{\mathrm{PD}}\right)$. Based on the order statistics theory, the CDF of $\gamma_{P}$ is expressed as

$$
F_{\gamma_{P}}(\gamma)=F_{\gamma_{\mathrm{PR}}}(\gamma) F_{\gamma_{\mathrm{PD}}}(\gamma)
$$

Let $m_{l}$ and $\Omega_{l}$ be fading severity and channel mean power parameters of the $l$-th channel coefficient $h_{l}$, i.e., $X_{l}$ follows gamma distribution with parameters $\left(m_{l}, \alpha_{l}^{-1}\right), \alpha_{l}=\frac{m_{l}}{\Omega_{l}}$, as

$$
\begin{aligned}
& f_{X_{l}}\left(x_{l}\right)=\frac{\alpha_{l}^{m_{l}}}{\Gamma\left(m_{l}\right)} x_{l}^{m_{l}-1} \exp \left(-\alpha_{l} x_{l}\right) \\
& F_{X_{l}}\left(x_{l}\right)=1-\exp \left(-\alpha_{l} x_{l}\right) \sum_{p=0}^{m_{1}-1} \frac{\alpha_{1}^{p} x_{1}^{p}}{p !}
\end{aligned}
$$

\section{PERFormance OF PRIMARY TRANSMISSION}

In this section, we present an exact closed-form expression for the CDF of the instantaneous SNR, $\gamma_{P}$, for the primary sub-system. Utilizing this outcome, we further derive exact closed-form expressions for the OP and the SER in the sequel.

From (7), the CDF of $\gamma_{\mathrm{PR}}$ is determined as

$$
F_{\gamma_{\mathrm{PR}}}(\gamma)=\int_{0}^{\infty} F_{X_{3}}\left(\gamma c x_{1}\right) f_{X_{1}}\left(x_{1}\right) d x_{1}
$$

Substituting (10) and (11) into (12), then applying [10, eq. (3.381.4)] to solve the remaining integral, $F_{\gamma_{\mathrm{PR}}}(\gamma)$ is formulated as

$$
F_{\gamma_{\mathrm{PR}}}(\gamma)=1-\sum_{p=0}^{m_{3}-1} \frac{1}{p !} \frac{\Gamma\left(m_{1}+p\right)}{\Gamma\left(m_{1}\right)} \frac{\alpha_{1}^{m_{1}} \alpha_{3}^{p} c^{p} \gamma^{p}}{\left(\alpha_{3} \gamma c+\alpha_{1}\right)^{\left(m_{1}+p\right)}}
$$

From (8), the CDF of $\gamma_{\mathrm{PD}}$ is given by

$$
F_{\gamma_{\mathrm{PD}}}(\gamma)=F_{X_{5}}\left(\frac{\gamma}{d}\right)=1-\exp \left(-\frac{\alpha_{5} \gamma}{d}\right) \sum_{q=0}^{m_{5}-1} \frac{\alpha_{5}^{q} \gamma^{q}}{d^{q} q !}
$$

Substituting (13) and (14) into (9), the CDF of the end-to-end SNR $\gamma_{P}$ is obtained as

$$
\begin{aligned}
& F_{\gamma_{P}}(\gamma)=1-\sum_{p=0}^{m_{3}-1} \frac{1}{p !} \frac{\Gamma\left(m_{1}+p\right)}{\Gamma\left(m_{1}\right)} \frac{\alpha_{1}^{m_{1}} \alpha_{3}^{p} c^{p} \gamma^{p}}{\left(\alpha_{3} \gamma c+\alpha_{1}\right)^{\left(m_{1}+p\right)}} \\
& -\exp \left(-\frac{\alpha_{5} \gamma}{d}\right) \sum_{q=0}^{m_{5}-1} \frac{\alpha_{5}^{q} \gamma^{q}}{d^{q} q !}+\sum_{p=0}^{m_{3}-1} \frac{1}{p !} \frac{\Gamma\left(m_{1}+p\right)}{\Gamma\left(m_{1}\right)} \\
& \times \frac{\alpha_{1}^{m_{1}} \alpha_{3}^{p} \gamma^{p} c^{p}}{\left(\alpha_{3} \gamma c+\alpha_{1}\right)^{\left(m_{1}+p\right)}} \exp \left(-\frac{\alpha_{5} \gamma}{d}\right) \sum_{q=0}^{m_{5}-1} \frac{\alpha_{5}^{q} \gamma^{q}}{d^{q} q !}
\end{aligned}
$$




\section{A. Outage Performance}

Outage probability of the primary system, $P_{\text {out }}^{P}$, is defined as the probability that the instantaneous SNR, $\gamma_{P}$, falls below a predefined threshold, $\gamma_{t h}$. This performance metric is found directly from the CDF of $\gamma_{P}$ given in (15) as follows:

$$
P_{\text {out }}^{P}=F_{\gamma_{P}}\left(\gamma_{t h}\right)
$$

\section{B. Symbol Error Rate}

In general, the SER of the primary system, $P_{E}^{P}$, can be given in terms of $F_{\gamma_{P}}(\gamma)$ as follows [11]:

$$
P_{E}^{P}=\frac{a_{1} \sqrt{b_{1}}}{2 \sqrt{\pi}} \int_{0}^{\infty} F_{\gamma_{P}}(\gamma) \gamma^{-\frac{1}{2}} \exp \left(-b_{1} \gamma\right) d \gamma
$$

where $a_{1}$ and $b_{1}$ are modulation parameters determined by the specific modulation scheme, e.g., $a_{1}=2$ and $b_{1}=\sin \left(\frac{\pi}{M}\right)^{2}$ for $M$-ary phase shift keying ( $M$-PSK). Substituting (15) into (17), we rewrite the expression for the SER as

$$
\begin{aligned}
& P_{E}^{P}=\frac{a_{1} \sqrt{b_{1}}}{2 \sqrt{\pi}} \int_{0}^{\infty} \gamma^{-\frac{1}{2}} \exp \left(-b_{1} \gamma\right) d \gamma-\frac{a_{1} \sqrt{b_{1}}}{2 \sqrt{\pi}} \sum_{p=0}^{m_{3}-1} \frac{1}{p !} \\
& \times \frac{\Gamma\left(m_{1}+p\right)}{\Gamma\left(m_{1}\right)} \frac{\alpha_{1}^{m_{1}}}{\alpha_{3}^{m_{1}} c^{m_{1}}} \int_{0}^{\infty} \frac{\gamma^{p-\frac{1}{2}} \exp \left(-b_{1} \gamma\right)}{\left(\gamma+\frac{\alpha_{1}}{\alpha_{3} c}\right)^{m_{1}+p}} d \gamma \\
& -\frac{a_{1} \sqrt{b_{1}}}{2 \sqrt{\pi}} \sum_{q=0}^{m_{5}-1} \frac{\alpha_{5}^{q}}{d^{q} q !} \int_{0}^{\infty} \exp \left(-\frac{\left(\alpha_{5}+d b_{1}\right) \gamma}{d}\right) \gamma^{q-\frac{1}{2}} d \gamma \\
& +\frac{a_{1} \sqrt{b_{1}}}{2 \sqrt{\pi}} \sum_{p=0}^{m_{3}-1} \frac{1}{p !} \frac{\Gamma\left(m_{1}+p\right)}{\Gamma\left(m_{1}\right)} \sum_{q=0}^{m_{5}-1} \frac{\alpha_{1}^{m_{1}}}{q ! \alpha_{3}^{m_{1}}} \frac{\alpha_{5}^{q}}{c^{m_{1}} d^{q}} \\
& \times \int_{0}^{\infty} \frac{\gamma^{p+q-\frac{1}{2}}}{\left(\gamma+\frac{\alpha_{1}}{\alpha_{3} c}\right)^{\left(m_{1}+p\right)} \exp \left(-\frac{\left(\alpha_{5}+d b_{1}\right) \gamma}{d}\right) d \gamma}
\end{aligned}
$$

Applying [10, eq. (3.381.4)] to solve the first and third integrals, and [12, eq. (2.3.6.9)] to simplify the second and fourth integral of (18), we obtain

$$
\begin{aligned}
& P_{E}^{P}=\frac{a_{1}}{2}-\frac{a_{1} \sqrt{b_{1}}}{2 \sqrt{\pi}} \sum_{p=0}^{m_{3}-1} \frac{1}{p !} \frac{\Gamma\left(m_{1}+p\right) \Gamma\left(p+\frac{1}{2}\right)}{\Gamma\left(m_{1}\right)} \frac{\alpha_{1}^{\frac{1}{2}}}{\alpha_{3}^{\frac{1}{2}}} \\
& \times \frac{1}{c^{\frac{1}{2}}} U\left(p+\frac{1}{2}, \frac{3}{2}-m_{1}, b_{1} \frac{\alpha_{1}}{\alpha_{3} c}\right)-\frac{a_{1} \sqrt{b_{1}}}{2 \sqrt{\pi}} \sum_{q=0}^{m_{5}-1} \frac{\alpha_{5}^{q}}{q !} \\
& \times d^{\frac{1}{2}} \frac{\Gamma\left(q+\frac{1}{2}\right)}{\left(\alpha_{5}+d b_{1}\right)^{q+\frac{1}{2}}}+\frac{a_{1} \sqrt{b_{1}}}{2 \sqrt{\pi}} \frac{1}{p !} \frac{\Gamma\left(m_{1}+p\right)}{\Gamma\left(m_{1}\right)} \sum_{q=0}^{m_{5}-1} \frac{1}{q !} \\
& \times \frac{\alpha_{5}^{q}}{d^{q}} \frac{\alpha_{1}^{q+\frac{1}{2}}}{\alpha_{3}^{q+\frac{1}{2}} c^{q+\frac{1}{2}}} \Gamma\left(p+q+\frac{1}{2}\right) U\left(p+q+\frac{1}{2}, p+q\right. \\
& \left.+\frac{3}{2}-m_{1}-p, \frac{\alpha_{5} \alpha_{1}+d b_{1} \alpha_{1}}{\alpha_{3} c d}\right)
\end{aligned}
$$

\section{Performance of Secondary Transmission}

In this section, an exact closed-form expression for the CDF of the instantaneous SNR $\gamma_{S}$ is first derived to further quantify the outage performance of the secondary system. Moreover, the CDF of a tight upper bound on $\gamma_{S}$ is also introduced to evaluate the SER of the secondary system.

\section{A. Outage Probability}

It can be seen from (6) that $\gamma_{S}$ is expressed as a complicated function of multiple independent RVs, i.e., $X_{i}$ with $i=1,2,3,4$; thus, the total probability theorem is utilized to obtain its CDF. Fixing the values of $X_{3}$ and $X_{4}$ as $X_{3}=x_{3}$ and $X_{4}=x_{4}$, we have the respective conditional CDF of $\gamma_{S}$, $F_{\gamma_{S}}\left(\gamma \mid x_{3}, x_{4}\right)$, as follows:

$$
\begin{aligned}
& F_{\gamma_{S}}\left(\gamma \mid x_{3}, x_{4}\right)=P\left\{\frac{X_{1} X_{2}}{a X_{2} x_{3}+b X_{1} x_{4}}<\gamma\right\} \\
& =\underbrace{F_{X_{2}}\left(b \gamma x_{4}\right)}_{I\left(\gamma \mid x_{4}\right)}+\underbrace{\int_{b \gamma x_{4}}^{\infty} F_{X_{1}}\left(\frac{a \gamma x_{2} x_{3}}{x_{2}-b \gamma x_{4}}\right) f_{X_{2}}\left(x_{2}\right) d x_{2}}_{J\left(\gamma \mid x_{3}, x_{4}\right)}
\end{aligned}
$$

It can be seen that $I(\gamma)$ is simply achieved by averaging the conditional CDF $I\left(\gamma \mid x_{4}\right)$ over the PDF of $X_{4}$ as $I(\gamma)=$ $\int_{0}^{\infty} I\left(\gamma \mid x_{4}\right) f_{X_{4}}\left(x_{4}\right) d x_{4}$. Using (10), (11) together with [10, eq. (3.381.4)], we have

$$
I(\gamma)=1-\frac{\alpha_{4}^{m_{4}}}{\Gamma\left(m_{4}\right)} \sum_{q=0}^{m_{2}-1} \frac{\alpha_{2}^{q} \gamma^{q} b^{q}}{q !} \frac{\Gamma\left(m_{4}+q\right)}{\left(\alpha_{2} \gamma b+\alpha_{4}\right)^{\left(m_{4}+q\right)}}
$$

In addition, the expression for $J\left(\gamma \mid x_{3}, x_{4}\right)$ is rewritten as

$$
\begin{aligned}
& J\left(\gamma \mid x_{3}, x_{4}\right) \\
& =\int_{0}^{\infty} F_{X_{1}}\left(\gamma a x_{3}+\frac{\gamma^{2} a b x_{4} x_{3}}{x_{2}}\right) f_{X_{2}}\left(x_{2}+\gamma b x_{4}\right) d x_{2}
\end{aligned}
$$

By substituting (10) and (11) into (22) and then utilizing the binomial theorem in [10, eq. (1.111)], i.e., $(a+b)^{n}=$ $\sum_{0}^{n} C_{k}^{n} x^{k} y^{n-k}$ where $C_{k}^{n}=\frac{n !}{k !(n-k) !}$ is the binomial coefficient, as well as the result of [10, eq. (3.471.9)] to solve the remaining integral of (22), an analytic expression for $J\left(\gamma \mid x_{3}, x_{4}\right)$ is found as

$$
\begin{aligned}
& J\left(\gamma \mid x_{3}, x_{4}\right)=1-F_{X_{2}}\left(\gamma b x_{4}\right)-2 \sum_{p=0}^{m_{1}-1} \sum_{q=0}^{p} \sum_{r=0}^{2 m_{2}-1} \frac{C_{q}^{p} C_{r}^{m_{2}-1}}{p ! \Gamma\left(m_{2}\right)} \\
& \times \alpha_{1}^{\frac{r+p+q+1}{2}} \alpha_{2}^{\frac{2 m_{2}-r+p-q-1}{2}} a^{\frac{r+p+q+1}{2}} b^{\frac{2 m_{2}+p-q-r-1}{2}} \gamma^{m_{2}+p} \\
& \times x_{3}^{\frac{r+p+q+1}{2}} x_{4}^{\frac{2 m_{2}+p-q-r-1}{2}} \exp \left(-\alpha_{1} \gamma a x_{3}\right) \exp \left(-\alpha_{2} \gamma b x_{4}\right) \\
& \times \mathcal{K}_{r-p+q+1}\left(2 \sqrt{\alpha_{1} \alpha_{2} \gamma^{2} a b x_{3}} \sqrt{x_{4}}\right)
\end{aligned}
$$

By integrating the product of $J\left(\gamma \mid x_{3}, x_{4}\right)$ and $f_{X_{4}}\left(x_{4}\right)$ over $x_{4}$, yields

$$
J\left(\gamma \mid x_{3}\right)=\int_{0}^{\infty} J\left(\gamma \mid x_{3}, x_{4}\right) f_{X_{4}}\left(x_{4}\right) d x_{4}
$$

Substituting (23) and (10) in (24), then applying [10, eq. (3.381.4)] and [10, eq. (6.643.3)] to tabulate the first and second integrals, respectively, the expression for $J\left(\gamma \mid x_{3}\right)$ is 
represented as

$$
\begin{aligned}
& J\left(\gamma \mid x_{3}\right)=\frac{\alpha_{4}^{m_{4}}}{\Gamma\left(m_{4}\right)} \sum_{q=0}^{m_{2}-1} \frac{\alpha_{2}^{q} \gamma^{q} b^{q}}{q !} \frac{\Gamma\left(m_{4}+q\right)}{\left(\alpha_{2} \gamma b+\alpha_{4}\right)^{\left(m_{4}+q\right)}} \\
& -\sum_{p=0}^{m_{1}-1} \sum_{q=0}^{p} \sum_{r=0}^{m_{2}-1} \frac{C_{q}^{p} C_{r}^{m_{2}-1} \Gamma\left(m_{4}+m_{2}+p-q-r-1\right)}{p ! \Gamma\left(m_{2}\right) \Gamma\left(m_{4}\right)} \\
& \times \Gamma\left(m_{4}+m_{2}\right) a^{\frac{r+p+q}{2}} b^{\frac{2 m_{2}-r+p-q-2}{2}} \alpha_{1}^{\frac{r+p+q}{2}} \alpha_{2}^{\frac{2 m_{2}-r+p-q-2}{2}} \\
& \times \alpha_{4}^{m_{4}} \frac{\gamma^{m_{2}+p-1} \exp \left(-\alpha_{1} \gamma a x_{3}\right)}{\left(\alpha_{4}+\alpha_{2} \gamma b\right)^{\frac{2 m_{4}+2 m_{2}+p-q-r-2}{2}}} \exp \left(\frac{\alpha_{1} \alpha_{2} \gamma^{2} a b x_{3}}{2\left(\alpha_{4}+\alpha_{2} \gamma b\right)}\right) \\
& \times x_{3}^{\frac{r+p+q}{2}} \mathcal{W}_{-\frac{2 m_{4}+2 m_{2}+p-q-r-2}{2}, \frac{r-p+q+1}{2}}\left(\frac{\alpha_{1} \alpha_{2} \gamma^{2} a b x_{3}}{\alpha_{4}+\alpha_{2} \gamma b}\right)
\end{aligned}
$$

Now, $J(\gamma)$ is obtained by integrating the product $J\left(\gamma \mid x_{3}\right) f_{X_{3}}\left(x_{3}\right)$ over $x_{3}$ as

$$
J(\gamma)=\int_{0}^{\infty} J\left(\gamma \mid x_{3}\right) f_{X_{3}}\left(x_{3}\right) d x_{3}
$$

Substituting (10) and (25) into (26) followed by utilizing [10, eq. (7.621.3)] to solve the integral (26), $J(\gamma)$ is now obtained as

$$
\begin{aligned}
& J(\gamma)=\frac{\alpha_{4}^{m_{4}}}{\Gamma\left(m_{4}\right)} \sum_{q=0}^{m_{2}-1} \frac{\alpha_{2}^{q} b^{q} \gamma^{q} \Gamma\left(m_{4}+q\right)}{q !\left(\alpha_{2} \gamma b+\alpha_{4}\right)^{\left(m_{4}+q\right)}}-\sum_{p=0}^{m_{1}-1} \frac{1}{p !} \sum_{q=0}^{p} C_{q}^{p} \\
& \times \sum_{r=0}^{m_{2}-1} C_{r}^{m_{2}-1} \frac{\Gamma\left(m_{4}+m_{2}+p-q-r-1\right) \Gamma\left(m_{4}+m_{2}\right)}{\Gamma\left(m_{2}\right) \Gamma\left(m_{4}\right) \Gamma\left(m_{3}\right)} \\
& \times \frac{\alpha_{1}^{r+q+1} \alpha_{2}^{m_{2}} \alpha_{3}^{m_{3}} \alpha_{4}^{m_{4}} a^{r+q+1} b^{m_{2}} \gamma^{m_{2}+r+q+1}}{\left(\alpha_{4}+\alpha_{2} \gamma b\right)^{m_{4}+m_{2}}\left(\alpha_{3}+\alpha_{1} \gamma a\right)^{m_{3}+r+q+1}} \Gamma\left(m_{3}+p\right) \\
& \times \frac{\Gamma\left(m_{3}+r+q+1\right)}{\Gamma\left(m_{3}+m_{4}+m_{2}+p\right)} F_{1}\left(m_{3}+r+q+1, m_{4}+m_{2}, m_{3}\right. \\
& \left.+m_{4}+m_{2}+p ; \frac{\alpha_{3} \alpha_{4}+\alpha_{2} \alpha_{3} \gamma b+\alpha_{1} \alpha_{4} \gamma a}{\alpha_{3} \alpha_{4}+\alpha_{2} \alpha_{3} \gamma b+\alpha_{1} \alpha_{4} \gamma a+\alpha_{1} \alpha_{2} \gamma^{2} a b}\right)
\end{aligned}
$$

It is straightforward to see that the CDF of $\gamma_{S}$ can be formulated by adding $I(\gamma)$ given in (21) and $J(\gamma)$ given in (27) together as (28) on the next page. As a result, the outage probability of the secondary network is obtained directly from (28) as $P_{\text {out }}^{S}=F_{\gamma_{S}}\left(\gamma_{t h}\right)$.

\section{B. Symbol Error Rate}

It is not feasible to evaluate the SER of the secondary transmission by using the CDF of $\gamma_{S}, F_{\gamma_{S}}(\gamma)$. Instead, here, a tightly bounded expression for $F_{\gamma_{S}}(\gamma)$ is adopted to quantify the SER performance as in [13, eq. (25)]. To be specific, the instantaneous SNR of the secondary transmission $\gamma_{S}$ is upper bounded as $\gamma_{S}^{U}=\min \left(\gamma_{1}, \gamma_{2}\right)$ where $\gamma_{1}=\frac{X_{1}}{a X_{3}}$ and $\gamma_{2}=\frac{X_{2}}{b X_{4}}$. Hence, $F_{\gamma_{S}^{U}}(\gamma)$ is written as

$$
F_{\gamma_{S}^{U}}(\gamma)=1-\left[1-F_{\gamma_{1}}(\gamma)\right]\left[1-F_{\gamma_{2}}(\gamma)\right]
$$

where $F_{\gamma_{1}}(\gamma)=\int_{0}^{\infty} F_{X_{1}}\left(\gamma a x_{3}\right) f_{X_{3}}\left(x_{3}\right) d x_{3}$ and $F_{\gamma_{2}}(\gamma)=$ $\int_{0}^{\infty} F_{X_{2}}\left(\gamma b x_{4}\right) f_{X_{4}}\left(x_{4}\right) d x_{4}$. Utilizing (10) and (11) along with
[10, eq. (7.621.3)] to simplify $F_{\gamma_{i}}(\gamma), i=1,2$, yields

$$
\begin{aligned}
& F_{\gamma_{1}}(\gamma)=1-\sum_{p=0}^{m_{1}-1} \frac{1}{p !} \frac{\Gamma\left(m_{3}+p\right)}{\Gamma\left(m_{3}\right)} \frac{a^{p} \alpha_{1}^{p} \alpha_{3}^{m_{3}} \gamma^{p}}{\left(\alpha_{1} \gamma a+\alpha_{3}\right)^{\left(m_{3}+p\right)}} \\
& F_{\gamma_{2}}(\gamma)=1-\sum_{q=0}^{m_{2}-1} \frac{1}{q !} \frac{\Gamma\left(m_{4}+q\right)}{\Gamma\left(m_{4}\right)} \frac{b^{q} \alpha_{2}^{q} \alpha_{4}^{m_{4}} \gamma^{q}}{\left(\alpha_{4}+\alpha_{2} \gamma b\right)^{\left(m_{4}+q\right)}}
\end{aligned}
$$

Substituting (30) and (31) into (29), $F_{\gamma_{S}^{U}}(\gamma)$ is given by

$$
\begin{gathered}
F_{\gamma_{S}^{U}}(\gamma)=1-\sum_{p=0}^{m_{1}-1} \frac{1}{p !} \frac{\Gamma\left(m_{3}+p\right)}{\Gamma\left(m_{3}\right)} \sum_{q=0}^{m_{2}-1} \frac{1}{q !} \frac{\alpha_{3}^{m_{3}} \alpha_{4}^{N_{4} m_{4}} a^{N_{3} m_{3}}}{\alpha_{1}^{N_{3} m_{3}} \alpha_{2}^{N_{4} m_{4}}} \\
\times \frac{b^{N_{4} m_{4}} \Gamma\left(m_{4}+q\right)}{\Gamma\left(m_{4}\right)} \frac{\gamma^{p+q}}{\left(\gamma+\frac{\alpha_{3}}{\alpha_{1} a}\right)^{m_{3}+p}\left(\gamma+\frac{\alpha_{4}}{\alpha_{2} b}\right)^{m_{4}+q}}
\end{gathered}
$$

By replacing $F_{\gamma_{S}^{U}}(\gamma)$ for $F_{\gamma_{P}}(\gamma)$ and the modulation parameters $\left(a_{2}, b_{2}\right)$ of the secondary transmission for $\left(a_{1}, b_{1}\right)$ in (17), the SER for the secondary network is now written as

$$
\begin{aligned}
& P_{E}^{S}=\frac{a_{2}}{2}-\frac{a_{2} \sqrt{b_{2}}}{2 \sqrt{\pi}} \sum_{p=0}^{m_{1}-1} \frac{1}{p !} \frac{\Gamma\left(m_{3}+p\right)}{\Gamma\left(m_{3}\right)} \sum_{q=0}^{m_{2}-1} \frac{1}{q !} \frac{\alpha_{3}^{m_{3}} \alpha_{4}^{m_{4}}}{\alpha_{1}^{m_{3}} \alpha_{2}^{m_{4}}} \\
& \times \frac{\Gamma\left(m_{4}+q\right)}{a^{m_{3}} b^{m_{4}} \Gamma\left(m_{4}\right)} \int_{0}^{\infty} \frac{\gamma^{p+q-\frac{1}{2}} \exp \left(-b_{2} \gamma\right)}{\left(\gamma+\frac{\alpha_{3}}{\alpha_{1} a}\right)^{m_{3}+p}\left(\gamma+\frac{\alpha_{4}}{\alpha_{2} b}\right)^{m_{4}+q}} d \gamma
\end{aligned}
$$

Since the integral in (33) is not represented as a tabulable form, we apply the partial fraction [10, eq. (3.326.2)] for the integral expression and then utilizing [12, eq. (2.3.6.9)] to simplify the remaining integrals as follows:

$$
\begin{aligned}
& P_{E}^{S}=\frac{a_{2}}{2}-\frac{a_{2} \sqrt{b_{2}}}{2 \sqrt{\pi}} \sum_{p=0}^{m_{1}-1} \frac{1}{p !} \frac{\Gamma\left(m_{3}+p\right)}{\Gamma\left(m_{3}\right)} \sum_{q=0}^{m_{2}-1} \frac{1}{q !} \frac{\alpha_{3}^{m_{3}} \alpha_{4}^{m_{4}}}{\alpha_{1}^{m_{3}} \alpha_{2}^{m_{4}}} \\
& \times \frac{\Gamma\left(m_{4}+q\right) \Gamma\left(p+q+\frac{1}{2}\right)}{a^{m_{3}} b^{m_{4}} \Gamma\left(m_{4}\right)}\left[\sum_{i=1}^{m_{3}+p} \kappa_{i}\left(\frac{\alpha_{3}}{\alpha_{1} a}\right)^{p+q+\frac{1}{2}-i}\right. \\
& \times U\left(p+q+\frac{1}{2}, p+q+\frac{3}{2}-i, b_{2} \frac{\alpha_{3}}{\alpha_{1} a}\right)+\sum_{j=1}^{m_{4}+q} \theta_{j} \\
& \left.\times\left(\frac{\alpha_{4}}{\alpha_{2} b}\right)^{p+q+\frac{1}{2}-j} U\left(p+q+\frac{1}{2}, p+q+\frac{3}{2}-j, \frac{b_{2} \alpha_{4}}{\alpha_{2} b}\right)\right]
\end{aligned}
$$

where

$$
\begin{gathered}
\kappa_{i}=\left.\frac{1}{\left(m_{3}+p-i\right) !} \frac{d^{m_{3}+p-i}\left(\gamma+\frac{\alpha_{4}}{\alpha_{2} b}\right)^{-m_{4}-q}}{d \gamma^{m_{3}+p-i}}\right|_{\gamma=-\frac{\alpha_{3}}{\alpha_{1} a}} \\
\theta_{j}=\left.\frac{1}{\left(m_{4}+q-j\right) !} \frac{d^{m_{4}+q-j}\left(\gamma+\frac{\alpha_{3}}{\alpha_{1} a}\right)^{-m_{3}-r}}{d \gamma^{m_{4}+q-j}}\right|_{\gamma=-\frac{\alpha_{4}}{\alpha_{2} b}}
\end{gathered}
$$

\section{NUMERICAL RESULTS AND DISCUSSION}

In this section, numerical results are provided to verify the analysis and to illustrate the applicable prospect of the proposed scheme in improving system performance and enhancing frequency efficiency. Assuming that this system exits in a shadowed urban environment, so the path loss for an 


$$
\begin{aligned}
& F_{\gamma_{s}}(\gamma)=1-\sum_{p=0}^{m_{1}-1} \frac{1}{p !} \sum_{q=0}^{p} C_{q}^{p} \sum_{r=0}^{m_{2}-1} C_{r}^{m_{2}-1} \frac{\Gamma\left(m_{4}+m_{2}\right) \Gamma\left(m_{4}+m_{2}+p-q-r-1\right)}{\Gamma\left(m_{2}\right) \Gamma\left(m_{4}\right) \Gamma\left(m_{3}\right)} \alpha_{1}^{r+q+1} \alpha_{2}^{m_{2}} \alpha_{3}^{m_{3}} \alpha_{4}^{m_{4}} a^{r+q+1} b^{m_{2}} \\
& \times \frac{\gamma^{m_{2}+r+q+1}}{\left(\alpha_{4}+\alpha_{2} \gamma b\right)^{m_{4}+m_{2}}\left(\alpha_{3}+\alpha_{1} \gamma a\right)^{N_{3} m_{3}+r+q+1}} \frac{\Gamma\left(m_{3}+r+q+1\right) \Gamma\left(m_{3}+p\right)}{\Gamma\left(m_{3}+m_{4}+m_{2}+p\right)} F_{1}\left(m_{3}+r+q+1, m_{4}+m_{2}, m_{3}+m_{4}\right. \\
& \left.+m_{2}+p ; \frac{\alpha_{3} \alpha_{4}+\alpha_{2} \alpha_{3} \gamma b+\alpha_{1} \alpha_{4} \gamma a}{\alpha_{3} \alpha_{4}+\alpha_{2} \alpha_{3} \gamma b+\alpha_{1} \alpha_{4} \gamma a+\alpha_{1} \alpha_{2} \gamma^{2} a b}\right)
\end{aligned}
$$

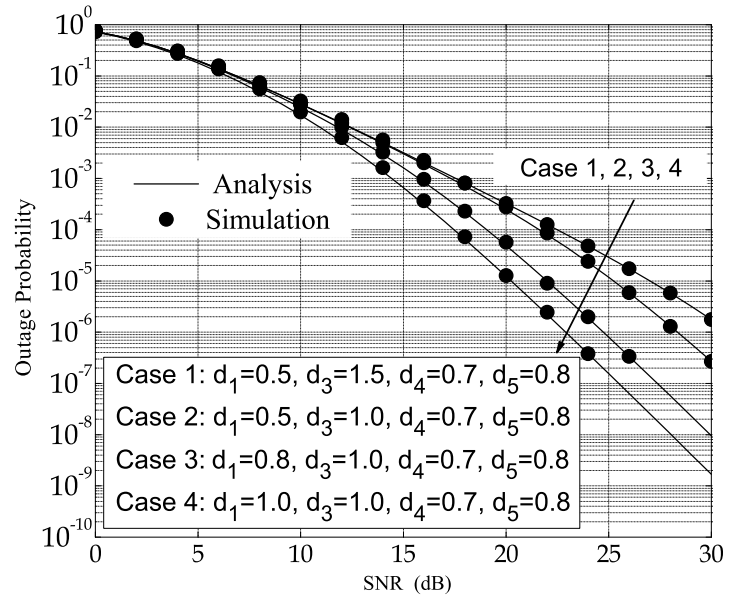

Fig. 2. Outage probability of the primary network with various distances of the links $\mathrm{SU}_{\mathrm{TX}} \rightarrow \mathrm{SU}_{\mathrm{R}}$ and $\mathrm{PU}_{\mathrm{TX}} \rightarrow \mathrm{SU}_{\mathrm{R}}$.

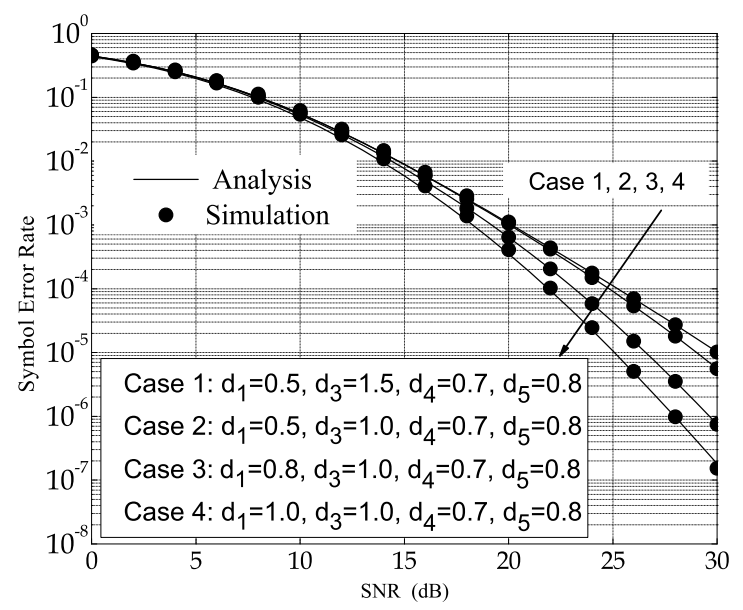

Fig. 3. Symbol error rate for 8-PSK of the primary network with various distances of the links $\mathrm{SU}_{\mathrm{TX}} \rightarrow \mathrm{SU}_{\mathrm{R}}$ and $\mathrm{PU}_{\mathrm{TX}} \rightarrow \mathrm{SU}_{\mathrm{R}}$.

arbitrary transmission distance $d$ may be modeled as $\left(\frac{d}{d_{0}}\right)^{-n}$ with path loss exponent $n=4$ where $d_{0}$ is the reference distance. For all considered scenarios, the fading severity parameters of all the links are set as $m_{i}=2, i=1, \cdots, 5$. Furthermore, the distances $\mathrm{SU}_{\mathrm{TX}} \rightarrow \mathrm{SU}_{\mathrm{R}}, \mathrm{SU}_{\mathrm{R}} \rightarrow \mathrm{SU}_{\mathrm{RX}}$, $\mathrm{PU}_{\mathrm{TX}} \rightarrow \mathrm{SU}_{\mathrm{R}}, \mathrm{SU}_{\mathrm{R}} \rightarrow \mathrm{PU}_{\mathrm{RX}}$, and $\mathrm{PU}_{\mathrm{TX}} \rightarrow \mathrm{PU}_{\mathrm{RX}}$ are normalized as $d_{1}, d_{2}, d_{3}, d_{4}$ and $d_{5}$, respectively. In this system model, the performance of the primary networks only depends on $d_{1}, d_{3}, d_{4}$, and $d_{5}$ and the performance of the secondary networks only depends on $d_{1}, d_{2}, d_{3}$ and $d_{4}$.

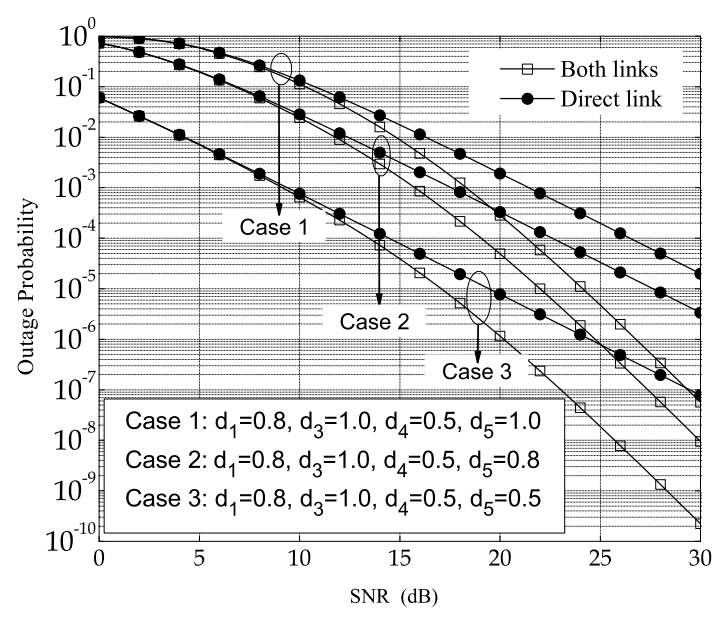

Fig. 4. A comparison of outage probability of the primary network with and without the help of the secondary relay.

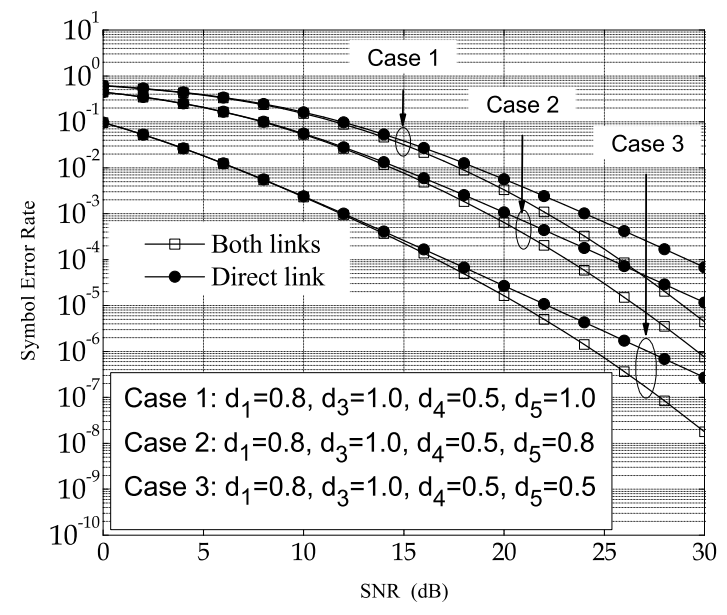

Fig. 5. A comparison of symbol error rate for 8-PSK of the primary network with and without the help of the secondary relay.

Fig. 2 and Fig. 3 depict the outage probability and SER of the primary network versus average $\mathrm{SNR}, P_{p} / N_{0}$, for different distances $\mathrm{SU}_{\mathrm{TX}} \rightarrow \mathrm{SU}_{\mathrm{R}}$ and $\mathrm{PU}_{\mathrm{TX}} \rightarrow \mathrm{SU}_{\mathrm{R}}$ while the distances $\mathrm{PU}_{\mathrm{TX}} \rightarrow \mathrm{SU}_{\mathrm{R}}$ and $\mathrm{PU}_{\mathrm{TX}} \rightarrow \mathrm{PU}_{\mathrm{RX}}$ is kept constant. Further, $P_{s}$ is set to $10 \mathrm{dBW}$ and $Q$ is set to $5 \mathrm{dBW}$. It can be seen from these figures that the outage probability and SER of the primary network are improved when $d_{1}$ increases. This performance enhancement can be attributed to the fact that when $d_{1}$ increases, there exits less interference from $\mathrm{SU}_{\mathrm{TX}}$ to the primary network. Furthermore, when $d_{3}$ decreases from 


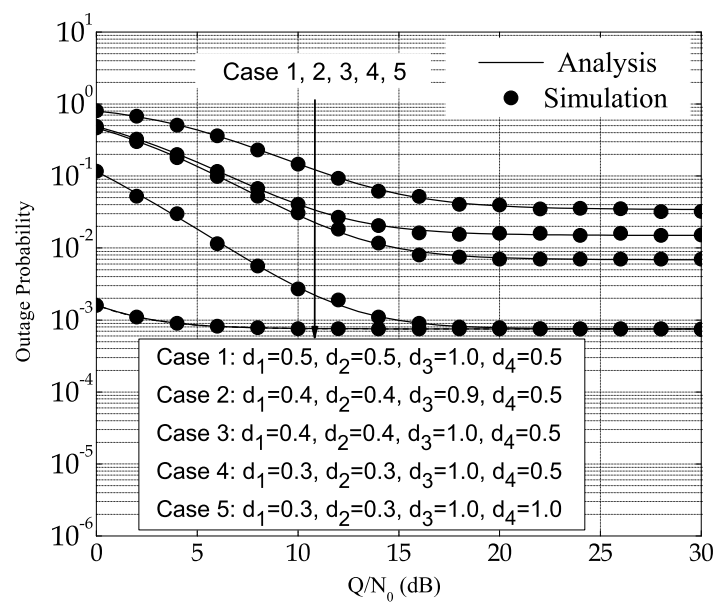

Fig. 6. Outage probability of the secondary network with various distances of the links $\mathrm{SU}_{\mathrm{TX}} \rightarrow \mathrm{SU}_{\mathrm{R}}, \mathrm{PU}_{\mathrm{TX}} \rightarrow \mathrm{SU}_{\mathrm{R}}$, and $\mathrm{SU}_{\mathrm{R}} \rightarrow \mathrm{SU}_{\mathrm{RX}}$.

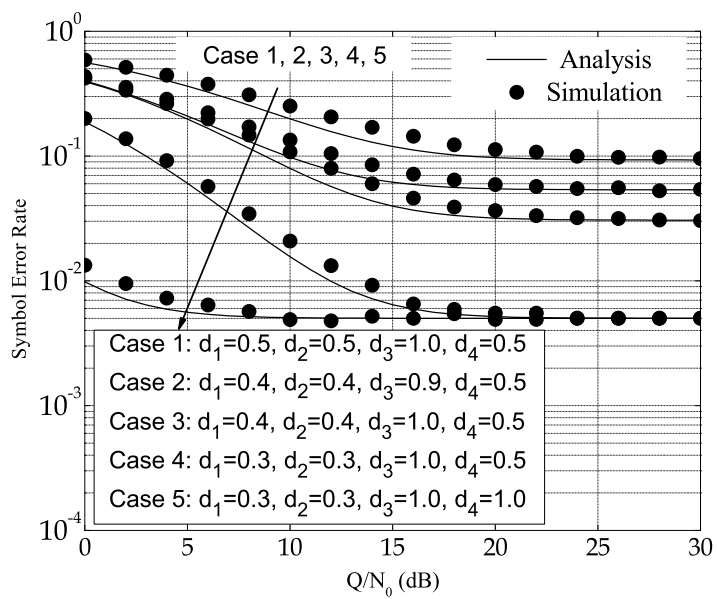

Fig. 7. Symbol error rate for 8-PSK of the secondary network with various distances of the links $\mathrm{SU}_{\mathrm{TX}} \rightarrow \mathrm{SU}_{\mathrm{R}}, \mathrm{PU}_{\mathrm{TX}} \rightarrow \mathrm{SU}_{\mathrm{R}}, \mathrm{SU}_{\mathrm{R}} \rightarrow \mathrm{SU}_{\mathrm{RX}}$.

$d_{3}=1.5$ in Case 1 to $d_{3}=1.0$ in Case 2 , the attenuation of the primary signal in the link from $\mathrm{PU}_{\mathrm{TX}}$ to $\mathrm{SU}_{\mathrm{R}}$ will become less severe. As a consequence, the outage probability and SER performance is improved.

Fig. 4 and Fig. 5 present a comparison between the outage probability and SER of the primary transmission for various distances $d_{5}$ from $\mathrm{SU}_{\mathrm{TX}}$ to $\mathrm{SU}_{\mathrm{RX}}$ with and without the help of the secondary relay (only the direct communication is adopted for the primary transmission). As expected, the performance when combining both the direct link and relaying link always outperforms that of having only a direct link. Therefore, in our model, the primary network performance is enhanced with the help of the secondary relay.

Fig. 6 and Fig. 7 depict the outage probability and SER of the secondary transmission for various $d_{1}, d_{2}, d_{3}$ and $d_{4}$. The transmit power of the primary network and secondary network are set equally to $P_{p}=P_{s}=10 \mathrm{dBW}$. Given the same distances $d_{3}$ and $d_{4}$, when the distances from $\mathrm{SU}_{\mathrm{TX}}$ to $\mathrm{SU}_{\mathrm{R}}$, $d_{1}$, and $\mathrm{SU}_{\mathrm{R}}$ to $\mathrm{SU}_{\mathrm{RX}}, d_{2}$, decrease, the outage probability and SER of secondary transmission are decreased. This is because attenuation due to path loss increases with distance. Moreover, by comparing Case 2 and Case 3, having the same values of $d_{1}, d_{2}$ and $d_{4}$, the better performance is achieved in Case 3 with larger value of $d_{3}$ because of less interference from PUTX to the secondary transmission.

Finally, when the distance from $\mathrm{SU}_{\mathrm{R}}$ to $\mathrm{PU}_{\mathrm{RX}}, d_{4}$, is sufficiently large such as in Case 5 of Fig. 6 and Fig. 7, the outage probability and SER of the secondary transmission are almost invariant with the increase of the interference threshold $Q$. This is due to the fact that the signal from the secondary transmission is severely attenuated over the large distance $d_{4}$ from $\mathrm{SU}_{\mathrm{R}}$ to $\mathrm{SU}_{\mathrm{RX}}$. Hence, the interference caused by the secondary transmission to $\mathrm{PU}_{\mathrm{RX}}$ is negligible. As a result, for any interference threshold, $Q$, this interference is always smaller than $Q$.

\section{CONCLUSIONS}

In this paper, we considered a system model for spectrum sharing where the AF cognitive relay assisted both the primary transmitter and the secondary transmitter in forwarding their signals. In particular, we derived exact closed-form expressions for the outage probability and SER of the primary transmission. Moreover, we also presented an exact closedform expression for the outage probability and a tight approximation for the SER of the secondary transmission. Our works show that in this system not only secondary transmission benefits from using the primary networks' frequency but also the primary transmission gain in performance by utilizing the relay of the secondary system. Finally, our analytical results are in close agreement with Monte Carlo simulations, which validates the presented analysis.

\section{REFERENCES}

[1] O. Simeone, J. Gambini, Y. Bar-Ness, and U. Spagnolini, "Cooperation and cognitive radio," in Proc. IEEE Int. Conf. Commun., Glasgow, Scotland, Jun. 2007, pp. 6511-6515.

[2] K. B. Letaief and W. Zhang, "Cooperative communications for cognitive radio networks," Proceedings of the IEEE, vol. 97, no. 5, pp. 878-893, May 2009.

[3] A. Ghasemi and E. Sousa, "Spectrum sensing in cognitive radio networks: requirements, challenges and design trade-offs," IEEE Commum. Magazine, vol. 46, no. 4, pp. 32-39, Apr. 2008.

[4] A. Louni and B. Khalaj, "Distributed beam-forming and power control in multi-relay underlay cognitive radio networks: a game-theoretical approach," in Proc. of Crowncom 2011, Osaka, Japan, Jun. 2011, pp. 71-75.

[5] Y. Han, A. Pandharipande, and S. Ting, "Cooperative decode-andforward relaying for secondary spectrum access," IEEE Trans. Wireless Commun., vol. 8, no. 10, pp. 4945-4950, Oct. 2009.

[6] T. Duong, V. Bao, G. Alexandropoulos, and H.-J. Zepernick, "Cooperative spectrum sharing networks with AF relay and selection diversity," Electron. Lett., vol. 47, no. 20, pp. 1149-1151, Sep. 2011.

[7] A. Yang, C. Xing, Z. Fei, and J. Kuang, "On the performance of joint relay selection and beamforming with limited feedback for AF cooperative networks," in Proc. IEEE Veh. technol. Conf., San Francisco, USA, Sep. 2011, pp. 1-5.

[8] J. Lee, H. Wang, J. G. Andrews, and D. Hong, "Outage probability of cognitive relay networks with interference constraints," IEEE Trans. Wireless Commun., vol. 10, no. 2, pp. 390-395, Feb. 2011.

[9] W. Jaafar, W. Ajib, and D. Haccoun, "A Novel Relay-Aided Transmission Scheme in Cognitive Radio Networks," in Proc. IEEE Global. Commun. Conf., Houston, USA, Dec. 2011, pp. 1-6.

[10] I. S. Gradshteyn and I. M. Ryzhik, Table of Integrals, Series, and Products, 7th ed. Academic Press, 2007.

[11] M. R. McKay, A. J. Grant, and I. B. Collings, "Performance analysis of MIMO-MRC in double-correlated Rayleigh environments," IEEE Trans. Commun., vol. 55, no. 3, pp. 497-507, Mar. 2007.

[12] A. P. Prudnikov, Y. A. Brychkov, and O. I. Marichev, Integrals and series Volume 1 elementary functions. New York: Gordon and Breach Science Publishers, 1986.

[13] M. D. Renzo, F. Graziosi, and F. Santucci, "A comprehensive framework for performance analysis of dual-hop cooperative wireless systems with fixed-gain relays over generalized fading channels," IEEE Trans. Wireless Commun., vol. 8, no. 10, pp. 5060-5074, 2009. 